\title{
Oligoribonuclease functions as a diribonucleotidase to bypass a key bottleneck in RNA degradation
}

Soo-Kyoung $\mathrm{Kim}^{1, *}$, Justin D. Lormand ${ }^{2, *}$, Cordelia A. Weiss ${ }^{1, *}$, Karin A. Eger ${ }^{2}$, Husan Turdiev, Asan Turdiev, Wade C. Winkler, ${ }^{1, \#}$, Holger Sondermann ${ }^{2, \#}$, Vincent T. Lee ${ }^{1, \#}$

${ }^{1}$ Department of Cell Biology and Molecular Genetics, University of Maryland, College Park, MD, USA

${ }^{2}$ Department of Molecular Medicine, College of Veterinary Medicine,

12 Cornell University, Ithaca, NY 14853, USA

13

${ }^{*}$ Authors contributed equally to this work.

17 Email: wwinkler@umd.edu, phone: 3014057780

18 Email: hs293@cornell.edu, phone: 6072533318

19 Email: vtlee@umd.edu, phone: 3014059397 


\section{Abstract}

24 Degradation of RNA polymers is a multistep process catalyzed by specific subsets of RNases. In

25 all cases, degradation is completed by exoribonucleases that recycle RNA fragments into 26 nucleotide monophosphate. In $\gamma$-proteobacteria, a group of up to eight 3 ' -5 ' exoribonucleases

27 have been implicated in RNA degradation. Oligoribonuclease (Orn) is unique among them as its

28 activity is required for clearing short RNA fragments, a function important for cellular fitness.

29 However, the mechanistic basis for this substrate selectivity remained unclear. Here we show

30 that Orn's activity as a general exoribonuclease has been vastly overestimated by demonstrating

31 that the enzyme exhibits a much narrower substrate preference for diribonucleotides. Co-crystal

32 structures of Orn with substrates reveal an active site optimized for diribonucleotides that does

33 not accommodate longer substrates. While other cellular RNases process oligoribonucleotides

34 down to diribonucleotide entities, our functional studies demonstrate that Orn is the one and

35 only diribonucleotidase that completes the final stage of the RNA degradation pathway.

36 Together, these results indicate that Orn is a dedicated diribonucleotidase that clears the

37 diribonucleotide pool that otherwise affects cellular physiology and viability. 


\section{Introduction}

Degradation of RNA is initiated by endonuclease-catalyzed cleavages; the resulting

oligoribonucleotide fragments are hydrolyzed to completion by a mixture of exoribonucleases for the

maintenance of cellular nucleotide pools ${ }^{1}$. Unlike the conserved machineries for the synthesis of

macromolecules, distinct sets of RNases are used by different organisms to degrade these

oligoribonucleotides. E. coli genomes encode eight 3'-5' exoribonucleases, namely polynucleotide phosphorylase, RNase II, D, BN, T, PH, R and oligoribonuclease (Orn) $)^{1,2}$. A subset of these enzymes recognizes structural features of the RNA substrate, such as in tRNA, while others act on unstructured polymers ${ }^{3}$.

As an exoribonuclease, Orn is unique for two reasons. First, orn is required for viability in many $\gamma$-proteobacteria, including $E . \operatorname{coli}^{4}$ and other organisms ${ }^{5,6}$, unlike all other known 3'-5',

exoribonucleases. This indicates that not all exoribonucleases have redundant functions despite acting

on the 3' end of RNA substrates and in several cases, including RNase R and RNase II, sharing an activity toward oligoribonucleotides ${ }^{7-10}$. Hence, Orn appears to catalyze a particularly important step in

RNA turnover. Second, Orn is a key enzyme in bacterial cyclic-di-GMP (c-di-GMP) signaling. The

nucleotide second messenger c-di-GMP is produced in bacteria in response to environmental cues and

controls a wide range of cellular pathways, including cell adhesion, biofilm formation, and virulence ${ }^{11-}$

14. Since the discovery of c-di-GMP over 30 years ago, it has been known that the signal is degraded

57 by a two-step process with a linear pGpG diribonucleotide intermediate ${ }^{15}$. While the enzyme for

58 linearizing c-di-GMP to pGpG was discovered early on ${ }^{16,17}$, the identity of the enzyme that degrades

59 pGpG remained elusive. Two recent studies showed that Orn is the primary enzyme that degrades

$60 \mathrm{pGpG}$ in Pseudomonas aeruginosa ${ }^{18,19}$. In an orn deletion strain, the accrual of linear pGpG has a

61 profound effect on cells. Specifically, the increase in pGpG inhibits upstream phosphodiesterases that 
62 degrade c-di-GMP, thereby triggering phenotypes associated with high cellular c-di-GMP levels.

63 However, the molecular basis of Orn's unique cellular functions in $\gamma$-proteobacteria that distinguishes

64 it from all other exoribonucleases remains unexplained.

65 Since its discovery over 50 years $\mathrm{ago}^{20}$, Orn has been presumed to degrade

66 oligoribonucleotides ${ }^{21-23}$. This notion largely derived from assays utilizing two types of substrates. In

67 one series of experiments, ${ }^{3} \mathrm{H}$ polyuridine (poly(U)) was incubated with Orn, other enzymes, or lysates

68 and analyzed by paper chromatography, which offers limited resolution overall ${ }^{21-23}$. In a second set of

69 experiments, Orn was incubated with oligoribonucleotides that had been tagged at their 5' terminus by

70 a large fluorophore. The products of this reaction were resolved by denaturing polyacrylamide gel

71 electrophoresis, thereby allowing for detection of products with one or more nucleotides removed from

72 the 3 ' terminus ${ }^{18,24-27}$. In both instances, it was concluded that Orn could processively degrade "short"

73 oligoribonucleotides. Yet it was not clear how Orn might selectively target "short" RNAs, rather than

74 simply binding to the penultimate sequence at the 3' termini of single-stranded RNA of any length.

Here, we sought to rigorously examine Orn's substrate preferences, revealing its unique

76 properties. To that end, we incubated Orn with $5,{ }^{32} \mathrm{P}$ end-labeled RNAs of varying lengths and analyzed the products of that reaction over time. Our data show that Orn exhibits a surprisingly narrow substrate preference for diribonucleotides. This finding is in stark contrast to the previous studies that described a broader substrate length range and that originally gave oligoribonuclease its name. We sought to understand this remarkable substrate selectivity of Orn by determining the crystal structures

81 of Orn in complex with pGpG and other linear diribonucleotides. These data reveal a structural basis

82 for the diribonucleotide preference and identify key residues for recognizing diribonucleotides.

83 Furthermore, we find that Orn is the only diribonucleotidase in P. aeruginosa. In addition, we show 84 that other diribonucleotides, in addition to $\mathrm{pGpG}$, can affect bacterial physiology. From this we 
propose a general model of RNA degradation, wherein a combination of exoribonucleases process oligoribonucleotides down to diribonucleotides and Orn completes RNA recycling by cleaving

87 diribonucleotides to nucleoside monophosphates. In this way, Orn occupies an important but discrete step in the overall RNA degradation pathway.

\section{Results and Discussion}

Orn functions as a diribonucleotidase in vitro. To understand the length preference of Orn, recombinant affinity-tagged Vibrio cholerae Orn $\left(\mathrm{Orn}_{V c}\right)$ was purified and tested biochemically. We used an established ligand-binding assay ${ }^{28}$ to determine the relative substrate affinities of Orn to $5^{\prime}-$ radiolabeled oligoribonucleotides that ranged from two to seven nucleotides in length. Unlike Cylabeled RNA used in several earlier studies ${ }^{18,24-27}$, radiolabeling with ${ }^{32} \mathrm{P}$ ensures that the substrate structure is unperturbed compared to native ligands. When spotted on nitrocellulose membranes, protein and protein-substrate complexes are sequestered at the point of application to the membrane, whereas unbound substrate diffuses due to radial capillary action ${ }^{28}$. By this assay, quantification of the diffusion zone reveals that $\operatorname{Orn}_{V c}$ exhibits the highest affinity for diribonucleotide $\left(K_{d}{ }^{\mathrm{pGpG}}=90-/+9\right.$ $\mathrm{nM}$ ), as compared to oligoribonucleotides of greater lengths (Table 1) ${ }^{19}$. Increase in the length to three or four residues reduces the affinity 7- or 10-fold, respectively. Substrates with 5 or more bases show a greater than 28-fold reduction in affinity compared to diribonucleotides. These results suggest that Orn has a strong preference for diribonucleotides over any other oligonucleotides.

105 that are unmodified at the 5' end, we incubated $\operatorname{Orn}_{V c}$ with $5^{\prime}-{ }^{32} \mathrm{P}$-radiolabeled oligoribonucleotides of 106 varying lengths in the presence of divalent cations that support catalysis. The products of these 
108 these electrophoresis conditions, the mononucleotides and oligonucleotides that were tested (between 2

109 and 7 nucleotides in length) can be resolved. The diribonucleotide substrate in this experiment was

$110 \mathrm{pGpG}(\mathrm{GG})$, whereas the longer oligoribonucleotides included an increasing number of adenine

111 nucleotides at the 5' end. This arrangement ensured that the same GG sequence was maintained at the

112 3' end while also avoiding stable G quadruplex formation that would be observed with RNAs

113 containing stretches of poly- $\mathrm{G}^{29}$. At substrate concentrations that far exceed enzyme concentration

114 (200:1) the diribonucleotide substrate was already fully processed to nucleoside monophosphates by 30

115 seconds (Figure 1A) ${ }^{19}$. In contrast, longer substrates (i.e., from 3-mers to 7-mers) were not processed at

116 their $3^{\prime}$ end, even at 30 minutes (Figures 1A and 1B). These results indicate that the length preference

117 for exoribonuclease activity of Orn on diribonucleotides is even greater than mere differences in

118 binding affinities. This strong substrate preference stands in stark contrast to previous studies arguing

119 Orn acts as a general exoribonuclease that cleaves oligoribonucleotides with 2 to 7 residues in 120 length ${ }^{18,21,22,24,25}$.

To determine if Orn can indeed cleave substrates longer than a diribonucleotide, the enzyme

122 was incubated with RNA substrates at a 1:1 molar ratio. Under these conditions, the diribonucleotide

123 substrate was completely processed to nucleoside monophosphates by the earliest time point, 20

124 seconds (Figure 1C and 1D). Orn $V_{c}$ also facilitated the degradation of the longer RNA substrates, but

125 only after significantly longer incubation times. For example, it required 10 minutes and 30 minutes to

126 fully degrade 3-mer and 4-mer RNAs, respectively (Figures 1C and 1D). Cleavage was reduced further

127 for longer RNAs; only 40-53\% of the 5-mer, 6-mer and 7-mer RNAs were processed to nucleoside

128 monophosphates at 30 minutes (Figures 1C and 1D), correlating with the weak affinities determined

129 for these substrates (Table 1). Of note, we observed a non-uniform distribution of degradation products

130 for the longer RNA substrates. Specifically, the diribonucleotide intermediate was never observed as a 
131 reaction intermediate for the longer RNAs. This reaction pattern indicates that RNAs of more than two

132 residues could accumulate, but that diribonucleotide RNAs were always rapidly processed to

133 nucleoside monophosphates. Together, these results indicate that Orn exhibits a strong substrate

134 preference for diribonucleotides over longer oligoribonucleotides - far greater than reported 135 previously $^{18,24}$.

137 Structure of Orn complexes with pGpG and other linear diribonucleotides. To elucidate the

138 molecular basis for these unique properties of Orn, we set out to gain a deeper understanding of the

139 enzyme's substrate specificity by determining the crystal structures of two representative homologs,

$140 \operatorname{Orn}_{V c}^{6}$ and the human REXO2 (also known as small fragment nuclease or $\mathrm{Sfn}^{30}$ ), bound to the

141 diribonucleotide pGpG (Table S1; Figures 2A and 2B). Superimposing the two structures indicates

142 their identical fold (rmsd of $0.75 \AA$ for the protomer; Figure S1A), which is preserved in the substrate-

143 free Orn structure determined previously (rmsd of $0.54 \AA$ for the protomer; Figure $\mathrm{S} 1 \mathrm{~A})^{31}$. The pGpG-

144 bound structures reveal a narrow active site that is lined by the conserved acidic residues of the

145 signature DEDD motif $\left(\mathrm{D}^{12}, \mathrm{E}^{14}\right.$, and $\mathrm{D}^{112}$ of $\mathrm{Orn}_{V c} ; \mathrm{D}^{15}, \mathrm{E}^{17}$, and $\mathrm{D}^{115}$ of REXO2) and the general base

$146 \mathrm{H}^{158}$ or His ${ }^{162}$ in $\mathrm{Orn}_{V c}$ or REXO2, respectively (Figures $2 \mathrm{~A}, 2 \mathrm{~B}$ and $\mathrm{S} 1 \mathrm{~B}$ ). In Orn ${ }_{V c}$, the bases of the

147 diribonucleotide buttress against aromatic residues $\mathrm{W}^{61}$ and $\mathrm{Y}^{129}$, the latter being contributed from the

148 second half-side of the dimeric enzyme. The corresponding residues, $\mathrm{W}^{64}$ and $\mathrm{Y}^{132}$, are conserved in

149 REXO2. Residue $\mathrm{L}^{18}$ in $\mathrm{Orn}_{V c}\left(\mathrm{~L}^{21}\right.$ in REXO2) wedges in-between the two bases. Most notably,

150 residues $\mathrm{S}^{108}, \mathrm{R}^{130}, \mathrm{~S}^{135}$ and the hydroxyl group on $\mathrm{Y}^{129}\left(\mathrm{~S}^{111}, \mathrm{R}^{133}, \mathrm{~S}^{138}\right.$, and $\mathrm{Y}^{132}$ in $\left.\mathrm{REXO} 2\right)$ form

151 hydrogen bonds with the 5' phosphate of $\mathrm{pGpG}$, capping the substrate. This phosphate cap creates a

152 major constriction of the active site, which is not observed in structurally related 3'-5' exoribonucleases

153 such as RNase T or ExoI (Figures 2C, S1C and S1D) ${ }^{32-34}$. RNase T and ExoI accommodate longer 
154 RNA substrates, facilitated by an expansive active site. The structural analysis correlates closely with

155 sequence conservation of the phosphate cap motif, which is strict in Orn homologs but divergent in 156 RNase T proteins (Figures 2D and S1B).

158 The phosphate cap is required for diribonucleotidase activity. To assess the impact on catalysis of

159 the phosphate cap in comparison to other active site residues identified in the structural analysis, we 160 introduced specific single-point mutations into $\operatorname{Orn}_{V c}$. Purified proteins were evaluated for $\mathrm{pGpG}$

161 degradation. Mutations of the central phosphate cap residue $\mathrm{R}^{130}$ to alanine led to complete loss of 162 catalytic activity, comparable to mutants in the DEDD active site motif (Figure $3 \mathrm{~A})^{19}$. We next asked 163 whether $\operatorname{Orn}_{V c}$ with specific point mutations could complement the deletion of orn in P. aeruginosa. P. 164 aeruginosa $\Delta$ orn accumulates pGpG that in turn inhibits c-di-GMP-specific phosphodiesterases ${ }^{18,19}$. As 165 a net result, c-di-GMP accumulates in these cells, an effect that is associated with a hyper-biofilm and

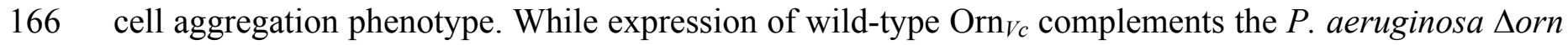
resulting in a dispersed culture, complementation with variants that carry mutations in either the active

168 site or phosphate cap residues results in cell aggregation indistinguishable from the $\Delta$ orn phenotype 169 (Figure 3B). Together, these experiments demonstrate that an intact phosphate cap is required for 170 enzyme function.

172 Interaction of Orn with substrates. Structures of $\operatorname{Orn}_{V c}$ (and REXO2) with different

173 diribonucleotides, including di-purine, di-pyrimidine and mixed substrates, revealed identical binding 174 poses (Figure S2). Additional hydrogen bonding between purine residues and Orn in the $3^{\prime}$ or $5^{\prime}$ 175 position correlates with a small, but detectable preference of $\operatorname{Orn}_{V c}$ for purine-containing substrates 176 (Figure 4). Together, the structural analysis uncovered Orn's mode of substrate binding, which is 
177 conserved from bacteria to humans and indicates a unique selection for linear diribonucleotides with a

178 5' phosphate.

179 Another distinguishing factor between Orn and other exoribonucleases with similar activities is

180 the apparent inhibition of Orn by $3^{\prime}$-phosphoadenosine $5^{\prime}$-phosphate (pAp), a metabolic byproduct of

181 sulfate assimilation that accumulates upon lithium poisoning of pAp-phosphatase ${ }^{25}$. DHH/DHHA-type

182 oligoribonucleases such as NrnA from Bacillus subtilis, Mycobacterium tuberculosis and Mycoplasma

183 pneumoniae are capable of dephosphorylating the pAp mononucleotide and their activity against

184 oligoribonucleotides is unaffected by $\mathrm{pAp}^{25,35}$. In contrast, $\mathrm{pAp}$ has been described as a competitive

185 inhibitor for E. coli Orn and the human REXO2 ${ }^{24}$. However, the oligo-cytosine RNAs used in these

186 prior studies were labeled at their 5' end with a bulky fluorescent dye moiety (usually a cyanine or Cy-

187 dye $)^{18,24}$; our aggregate data now indicate that these RNAs represent suboptimal substrates for Orn,

188 given its stark requirement for a simple 5' phosphate cap (Figure 2). This labeling strategy is therefore

189 likely to incompletely assess native Orn activity by underestimating diribonucleotidase activity while

190 also overestimating the effect of competitive inhibitors. Revisiting pAp binding to Orn, we show here

191 that $\operatorname{Orn}_{V_{c}}$ does not interact with radiolabeled pAp in the binding assay that was used to quantify

192 oligoribonucleotide interactions with Orn (Figure S3). Furthermore, unlabeled pAp failed to

193 competitively inhibit degradation of radiolabeled pGpG to GMP (Figure S3).

195 Orn is the only diribonucleotidase in $\boldsymbol{P}$. aeruginosa. All available literature assumes Orn is a 3'-5'

196 exoribonuclease that is responsible for processing of short (between 2-7) oligoribonucleotides. Yet our

197 structural and biochemical data suggest that the enzyme exhibits such a striking preference for

198 diribonucleotide substrates that the in vivo function of Orn as a general exoribonuclease should be

199 reconsidered. Therefore, we developed experimental conditions to measure Orn's activity in cellular 
extracts. The orn gene is required for viability in most $\gamma$-proteobacteria, including Vibrio cholerae;

201 however, it is not essential for growth of $P$. aeruginosa under most conditions ${ }^{18,19}$. Therefore, lysates

202 were generated from P. aeruginosa strains, including parental PA14, $\Delta$ orn, and $\Delta$ orn complemented 203 with rrn $_{V c} .5^{\prime}-{ }^{32} \mathrm{P}$-radiolabeled 2-mer or 7-mer RNA was then added to each of these lysates (Figure 5).

204 Aliquots of the mixtures were removed and analyzed by urea denaturing 20\% PAGE at varying time 205 intervals. Extracts from parental PA14 digested the entire radiolabeled diribonucleotide in less than 5 206 minutes (Figure 5A). In contrast, lysates from strains lacking Orn failed to show any signs of 207 diribonucleotidase activity even at longer time points. Diribonucleotidase activity in the lysates could 208 be restored by ectopic expression of orn from a self-replicating plasmid or by addition of purified Orn 209 (Figure 5A). These data confirm that cellular Orn is required for degrading diribonucleotides and no 210 other cellular RNase of $P$. aeruginosa can substitute for Orn activity.

211 When the ${ }^{32} \mathrm{P}-7-\mathrm{mer}$ RNA substrate was incubated in extracts from parental PA14 it was 212 digested to a ladder of degradative intermediates including 6-mer, 5-mer, 4-mer and 3-mer RNAs 213 (Figure 5B). Of note, while these intermediates and the final mononucleotide product accumulated 214 over time, the 2-mer intermediate was never observed over the time course. In contrast, lysates from 215 the $\Delta$ orn mutant specifically accrued the diribonucleotide intermediate with no apparent production of 216 its mononucleotide products. Ectopic expression of plasmid-borne orn restored the degradation of the 217 7-mer to mononucleotides and the diribonucleotide intermediate could no longer be observed. 218 Furthermore, the diribonucleotide intermediate that accumulated in the $\Delta$ orn lysate was fully processed 219 upon addition of purified Orn protein (Figure 5B). Together, these results show that P. aeruginosa 220 accumulates a bottleneck of diribonucleotide intermediates in a $\Delta o r n$ background, which is only 221 resolved upon addition of Orn. Degradation of RNA fragments with 3 or more residues by Orn is 222 negligible in a cellular context, considering that $\Delta$ orn lysates preserve nuclease activities for the 
223 processing of RNAs down to diribonucleotides. From these aggregate data we propose that Orn

224 functions not as an oligoribonuclease as stated in the literature but instead functions as a specialized 225 ribonuclease of diribonucleotide substrates (i.e., a 'diribonucleotidase').

227 The diribonucleotide pool affects $\boldsymbol{P}$. aeruginosa growth in addition to c-di-GMP. We noticed that 228 PA14 $\Delta$ orn had reduced growth on agar plates that is reminiscent of a small colony variant (SCV) 229 phenotype (Figure 6) ${ }^{36}$. The SCV phenotype has been attributed previously to increased c-di-GMP 230 levels $^{37}$. C-di-GMP binds to FleQ and activates transcription of the pel operon ${ }^{38,39}$. In addition, c-di231 GMP binds to the c-di-GMP-receptor PelD to increase the biosynthesis of the pel exopolysaccharide ${ }^{40}$, 232 which enhances cell aggregation leading to a compact SCV morphology. Previous reports had shown that $P$. aeruginosa $\Delta$ orn is unable to clear $\mathrm{pGpG}$, which results in the elevation of c-di-GMP signaling 234 and pel-dependent cell aggregation and biofilm mass ${ }^{18,19}$.

To determine whether the functional impact of diribonucleotide build-up is due specifically to an increased pGpG level and its effect on c-di-GMP, we asked whether the SCV formation in the $\Delta$ orn mutant is dependent on c-di-GMP processes. First, we confirmed that SCV formation in PA14 can be induced by elevated c-di-GMP levels through overexpression of diguanylate cyclases, such as PA1107, PA1120 and WspR (Figure 6A) ${ }^{37,41-43}$. As expected, the effect of c-di-GMP is due to increased 240 production of the pel exopolysaccharide and biofilm formation since the PA14 $\Delta$ pelA mutant 241 maintained normal colony morphology even when these diguanylate cyclases were overexpressed 242 (Figure 6A). To determine whether the increase in the pool of pGpG and c-di-GMP is the only reason 243 for small-colony growth in the $\Delta$ orn mutant, a $\Delta$ orn $\Delta p e l$ double mutant was tested. When $\Delta$ orn $\Delta p e l$ 244 was grown on agar, it also had an apparent SCV phenotype (Figure 6B). Complementation of $\Delta$ orn and $245 \Delta$ orn $\Delta p e l$ with active alleles of orn restored normal colony morphology, whereas inactive orn alleles 
246 failed to complement (Figure S4). In every case, the colony morphology was the same between $\Delta$ orn

247 and $\Delta$ orn $\Delta p e l$ indicating a second pathway that can restrict colony growth, but in this case

248 independent of pel and c-di-GMP. These results indicate that increased pools of one or more of the

249 diribonucleotides function to cause small-colony growth in $\Delta o r n$ in addition to the actions of pGpG on

250 c-di-GMP signaling.

251

252 Conclusion

Orn is unique amongst exoribonucleases because it is essential in some $\gamma$-proteobacteria and is 254 required to degrade the pGpG intermediate in c-di-GMP signaling. Yet the molecular and structural 255 requirements for Orn were unknown. Our studies reveal that Orn is a dedicated diribonucleotidase in 256 cells. This appears to be driven by a catalytic site that is restricted by a cap that mediates multiple 257 interactions with the 5' phosphate of diribonucleotide substrates. This constriction prevents longer 258 substrates from binding with high affinity, rendering them poor substrates for catalytic cleavage. The 259 discovery that Orn acts as a dedicated diribonucleotidase indicates that this activity clears a specific 260 diribonucleotide bottleneck in global RNA degradation (Figure 7). Prior studies have shown that orn 261 depletion can lead to accumulation of diribonucleotides and longer oligoribonucleotides in some 262 cellular backgrounds ${ }^{4}$. The increase in oligoribonucleotides longer than dimers in cells may occur 263 through feedback inhibition of other enzymes in the RNA degradation pathway, in analogy to the 264 impact of pGpG on c-di-GMP-degrading phosphodiesterases and c-di-GMP signaling ${ }^{18,19}$. Whether the 265 effect of diribonucleotides on essentiality is due to general processes such as RNA degradation, altered 266 transcription $^{44}$, specific interactions with essential proteins, or a combination of these remains to be 267 evaluated in this context. Our studies therefore reveal a key step in RNA degradation, the enzymatic 

not certified by peer review) is the author/funder. All rights reserved. No reuse allowed without permission.

268 cleavage of diribonucleotides into mononucleotides, and set the stage to address how diribonucleotide 269 accumulation is detrimental to cell survival. 


\section{Methods}

275 Cloning, protein expression and purification. Orn genes from Vibrio cholerae O1 El Tor VC0341 276 (residues 1-181) and Homo sapiens REXO2 (residues 33-237) were synthesized by Geneart (Life 277 Technologies). Genes were cloned by ligation between BamHI and NotI sites of a modified pET28a 278 vector (Novagen) yielding N-terminally His 6 -tagged small ubiquitin-like modifier (SUMO) fusion proteins cleavable by recombinant Ulp-1 protease.

Orn proteins were overexpressed in E. coli BL21 T7 Express cells (New England Biolabs). Fresh transformants were grown in Terrific Broth (TB) supplemented with $50 \mathrm{ug} / \mathrm{mL}$ kanamycin at $37^{\circ} \mathrm{C}$ to an $\mathrm{OD}_{600} \sim 1.0$, at which point the temperature was reduced to $18^{\circ} \mathrm{C}$ and expression was induced by addition of $0.5 \mathrm{mM}$ IPTG. Cells were harvested after $16 \mathrm{hr}$ of expression by centrifugation and resuspended in a minimal volume of Ni-NTA binding buffer $(25 \mathrm{mM}$ Tris-Cl, $500 \mathrm{mM} \mathrm{NaCl}, 20$ $\mathrm{mM}$ imidazole, $\mathrm{pH} 8.5$ ) followed by flash freezing in liquid nitrogen.

Cells were thawed and lysed by sonication. Cell debris was removed by centrifugation and clarified soluble lysate was incubated with Ni-NTA resin (Qiagen) pre-equilibrated with Ni-NTA binding buffer. Following one hour of binding, the resin was washed three times with 10 column volumes of Ni-NTA binding buffer, and then eluted with six column volumes of Ni-NTA elution buffer ( $25 \mathrm{mM}$ Tris-Cl, $500 \mathrm{mM} \mathrm{NaCl}, 350 \mathrm{mM}$ imidazole, $\mathrm{pH}$ 8.5). Eluates were buffer exchanged into gel filtration buffer ( $25 \mathrm{mM}$ Tris-Cl, $150 \mathrm{mM} \mathrm{NaCl}, \mathrm{pH}$ 7.5) via HiPrep $26 / 10$ desalting column (GE Healthcare), followed by overnight incubation with Ulp-1 to cleave off the His ${ }_{6}$-tagged SUMO moiety. Untagged Orn proteins were recovered in the flow-through of a HisTrap Ni-NTA column (GE Healthcare), separated from $\mathrm{His}_{6}$-SUMO, uncleaved proteins and His ${ }_{6}$-tagged Ulp-1. Orn was concentrated by Amicon Ultra 10K concentrator prior to loading onto a HiLoad 16/60 Superdex 200 gel filtration column (GE Healthcare) equilibrated in gel filtration buffer. Fractions containing Orn were pooled and concentrated to $100 \mathrm{mg} / \mathrm{mL}$, frozen in liquid nitrogen, and stored at $-80^{\circ} \mathrm{C}$.

Protein Crystallography. REXO2-RNA and $\mathrm{Orn}_{V c}$-RNA complexes (pGpG and pApA from BioLog Life Science Institute, other nucleotides from GE Healthcare Dharmacon) were formed prior to crystallization by mixing a 1:2 molar ratio of Orn:RNA in gel filtration buffer, followed by incubation for $30 \mathrm{~min}$ at the crystallization temperature. Orn-RNA complexes $(10-30 \mathrm{mg} / \mathrm{ml})$ were crystallized via hanging-drop vapor diffusion by mixing equal volumes $(0.8 \mu \mathrm{l})$ of sample with reservoir solution. 
$17 \%$ polyethylene glycol 3350 , and $20 \%$ xylitol. Crystals were flash-frozen in liquid nitrogen. REXO2 crystals grew at $4{ }^{\circ} \mathrm{C}$, using a reservoir comprised of $0.2 \mathrm{M}$ sodium malonate (pH 5.5) and 15-20\% polyethylene glycol 3350. REXO2 crystals were soaked in cryoprotectant of reservoir solution supplemented with $25 \%$ glycerol prior to flash freezing with liquid nitrogen. All crystals were stored in liquid nitrogen. Data were collected by synchrotron radiation at $0.977 \AA$ on frozen crystals at $100 \mathrm{~K}$ at beamline F1 of the Cornell High Energy Synchrotron Source (CHESS). Diffraction data sets were processed using XDS, Pointless, and Scala ${ }^{45,46}$. The initial structures were solved by Molecular Replacement using the software package Phenix ${ }^{47}$ and the unpublished coordinates of E. coli Orn (PDB: 2igi) as the search model. Manual model building and refinement were carried out with Coot ${ }^{48}$ and Phenix, respectively. Illustrations were prepared in Pymol (Version 2.2.0, Schrodinger, LLC). All software packages were accessed through SBGrid ${ }^{49}$. Crystallographic statistics are shown in Table S1.

Site-directed mutagenesis. To create the necessary mutants of $V c 0341 \mathrm{orn}$, mutations were generated by using the Q5 Site-Directed Mutagenesis Kit (New England Biolabs).

Labeling of RNAs. 5' un-phosphorylated RNAs were purchased from TriLink Biotechnologies or Sigma. Each RNA was subjected to radioactive end-labeling or non-radioactive phosphorylation by T4 Polynucleotide Kinase (New England Biolabs). Each RNA was subjected to phosphorylation with equimolar concentrations of either ${ }^{32} \mathrm{P}-\gamma$-ATP or ATP, T4 PNK, and 1X T4 PNK Reaction Buffer. Reactions comprising a final concentration of either $0.5 \mu \mathrm{M}$ radiolabeled RNA or $2.0 \mu \mathrm{M}$ phosphorylated RNA were incubated at $37^{\circ} \mathrm{C}$ for 40 minutes, followed by heat inactivation of T4 PNK at $65^{\circ} \mathrm{C}$ for 20 minutes.

Protein expression and purification for biochemical assays. E. coli T7Iq strains harboring expression vector pVL847 expressing an His-MBP-Orn and His-MBP-Orn mutants from $V$. cholerae were grown overnight, subcultured in M9 fresh media supplemented with $15 \mu \mathrm{g} / \mathrm{ml}$ gentamicin and grown to approximately $\mathrm{OD}_{600} 0.5 \sim 1.0$ at $30^{\circ} \mathrm{C}$. Expression was induced with $1 \mathrm{mM}$ IPTG for 4 hours. Induced bacteria were collected by centrifugation and resuspended in $10 \mathrm{mM}$ Tris, pH 8, $100 \mathrm{mM}$ $\mathrm{NaCl}$, and $25 \mathrm{mM}$ imidazole. After addition of DNase, lysozyme, and PMSF, bacteria were lysed by 
separation over a Ni-NTA column. Purified proteins were dialyzed overnight against $10 \mathrm{mM}$ Tris, $\mathrm{pH}$ $8,100 \mathrm{mM} \mathrm{NaCl}$, and 50\% (vol/vol) glycerol, aliquoted, and frozen at $-80{ }^{\circ} \mathrm{C}$.

Preparation of whole cell lysates. Overnight cultures of $P$. aeruginosa PA14 WT, Aorn mutant, or complemented strains were subcultured into fresh media with antibiotic and 1mM IPTG, grown at 37 ${ }^{\circ} \mathrm{C}$ with shaking. All bacteria samples were collected by centrifuge and resuspended in $100 \mathrm{mM} \mathrm{KCl,} 5$ $\mathrm{mM} \mathrm{MgCl}$, and $100 \mathrm{mM}$ Tris, $\mathrm{pH} \mathrm{8,} \mathrm{also} \mathrm{supplemented} \mathrm{with} \mathrm{lysozyme,} \mathrm{DNase,} \mathrm{and} \mathrm{PMSF} \mathrm{and} \mathrm{stored}$ at $-80{ }^{\circ} \mathrm{C}$.

Oligoribonuclease cleavage reactions. Phosphorylated RNA $(1.0 \mu \mathrm{M})$, including trace amounts of radiolabeled substrate, was subjected to cleavage by either $5.0 \mathrm{nM}$ or $1.0 \mu \mathrm{M}$ purified Orn at room temperature. These reactions were in the presence of $10 \mathrm{mM}$ Tris- $\mathrm{HCl} \mathrm{pH} 8.0,100 \mathrm{mM} \mathrm{NaCl}$, and 5 $\mathrm{mM} \mathrm{MgCl}_{2}$. At the appropriate times, aliquots of the reaction were removed and quenched in the presence of $150 \mathrm{mM}$ EDTA on ice. Aliquots were resolved by $20 \%$ urea-denaturing polyacrylamide gel electrophoresis (PAGE). The gel was imaged using Fujifilm FLA-7000 phosphorimager (GE). The intensity of the radiolabeled nucleotides was quantified using Fujifilm Multi Gauge software v3.0. Activity of whole cell lysates against ${ }^{32} \mathrm{P}$-labeled oligoribonucleotide substrates was measured by the reactions were performed at room temperature in reaction buffer $(10 \mathrm{mM}$ Tris, $\mathrm{pH} 8,100 \mathrm{mM} \mathrm{NaCl}$, and $5 \mathrm{mM} \mathrm{MgCl}_{2}$ ). At indicated time, the reaction stopped by the addition of $0.2 \mathrm{M}$ EDTA and heated at $98{ }^{\circ} \mathrm{C}$ for $5 \mathrm{~min}$. Samples were separated on $20 \%$ urea-denaturing PAGE and analyzed as indicated above.

DRaCALA measurement of dissociation constants. To measure $\mathrm{K}_{\mathrm{D}}$, serial dilutions of purified His-

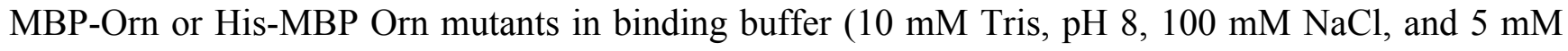
$\mathrm{CaCl}_{2}$ ) were mixed with radiolabeled nucleotides, applied to nitrocellulose sheets, dried, imaged and $\mathrm{K}_{\mathrm{D}}$ values were calculated as described previously ${ }^{28,50}$.

Aggregation assay. A colony of each strain of $P$. aeruginosa grown in LB agar plates supplemented 
366 for 18 22 hours. Culture tubes were allowed to settle at room temperature for 10 min and 367 photographed.

368

369 Data deposition. The atomic coordinates and structure factors have been deposited in the Protein Data

370 Bank, www.rcsb.org (PDB ID codes 6N6A, 6N6C, 6N6D, 6N6E, 6N6F, 6N6G, 6N6H, 6N6I, 6N6J, 371 and 6N6K).

372

\section{Acknowledgement}

374 This work was supported by the NIH via grant R01GM123609 (to H.S.), R01AI110740 375 (V.T.L.), Cystic Fibrosis Foundation (CF Foundation) LEE16G0 (V.T.L.) and National Science 376 Foundation (NSF) MCB1051440 (W.C.W.). C.A.W. was supported in part by the National Institutes of 377 Health $(\mathrm{NIH})$ training grant T32-GM080201. CHESS is supported by the NSF \& NIH/NIGMS via 378 NSF award DMR-1332208, and the MacCHESS resource is supported by NIH/NIGMS award 379 GM103485. 
Table 1. Quantitative measurement of length-dependent oligoribonucleotide affinities.

Substrate $^{a}$ Dissociation constant $K d(\mathrm{nM})^{\mathrm{b}}$

GG

$A G G$ $90-/+9$

AAGG

$630-/+80$

AAAGG

$890-/+90$

AAAAGG

$2,560-/+170$

AAAAAGG

$3,830-/+370$

A were labeled with ${ }^{32} \mathrm{P}$ at their 5 ' $3,750-1+530$

${ }^{a}$ : RNAs were labeled with ${ }^{32} \mathrm{P}$ at their 5 ' end. ${ }^{\mathrm{b}}$ : Affinities were measured using an established binding assay for radiolabeled RNAs ${ }^{28,50}$. 
Figure and Legends

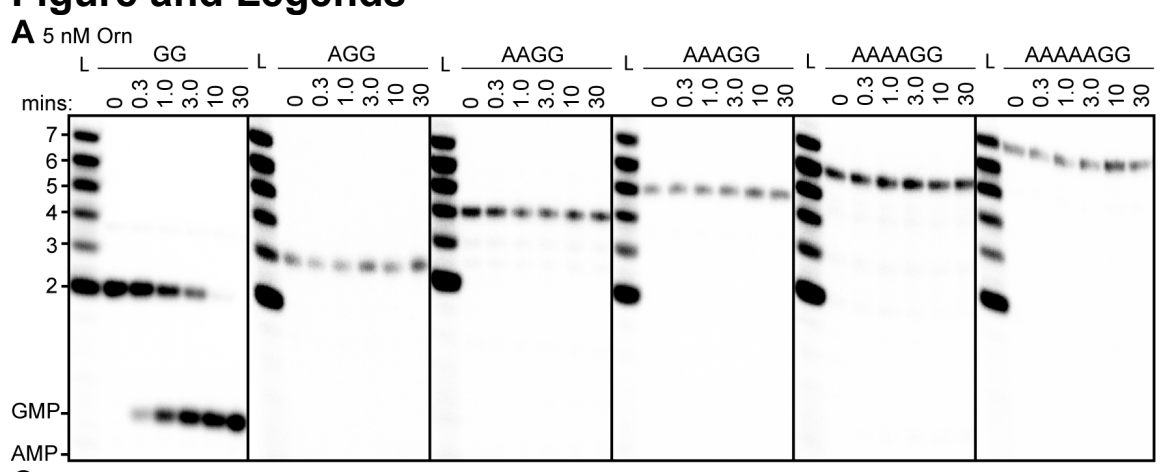

C $1,000 \mathrm{nM}$ Orn

387

388

389

390

391

392

393

394

395

396

397 average and SD of three independent experiments (B, D).
B

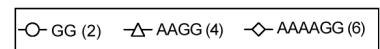
$-\square$ AGG (3) $\rightarrow$-AAAGG (5) $\sim$ - AAAAAGG (7)

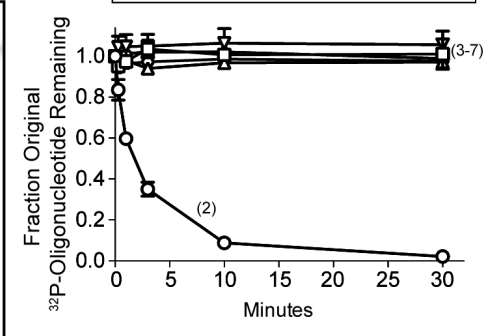

D

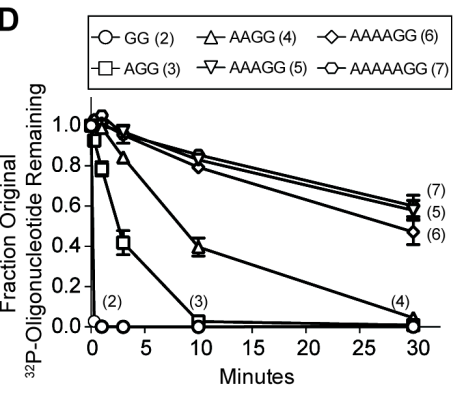

Figure 1. Orn has a stark preference for diribonucleotide cleavage in vitro. RNA nucleotides 2 to 7 residues in length $\left(1 \mu \mathrm{M}\right.$, containing the corresponding ${ }^{32} \mathrm{P}$-labeled RNA tracer) were each subjected to cleavage over time with $5 \mathrm{nM}(\mathrm{A}, \mathrm{B})$ or 1,000 $\mathrm{nM}$ Orn (C, D). Aliquots of each reaction were stopped at indicated times, and assessed by denaturing $20 \%$ polyacrylamide gel electrophoresis (A, C). Quantification of the intensities of bands corresponding to the amount of uncleaved initial oligonucleotide over time are plotted as the 

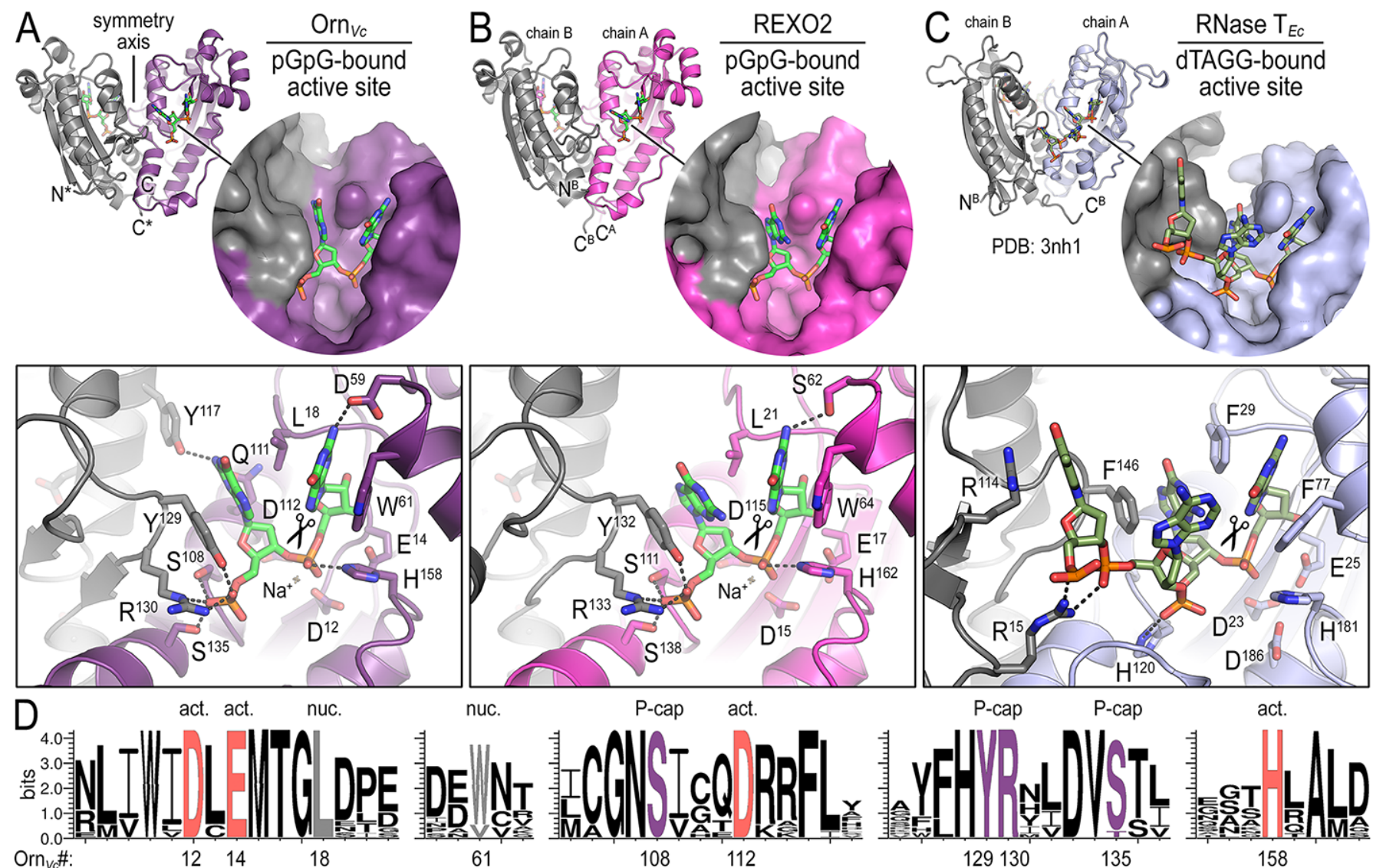

nuc.

P-cap act.

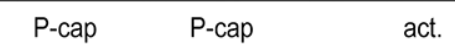

\section{.}
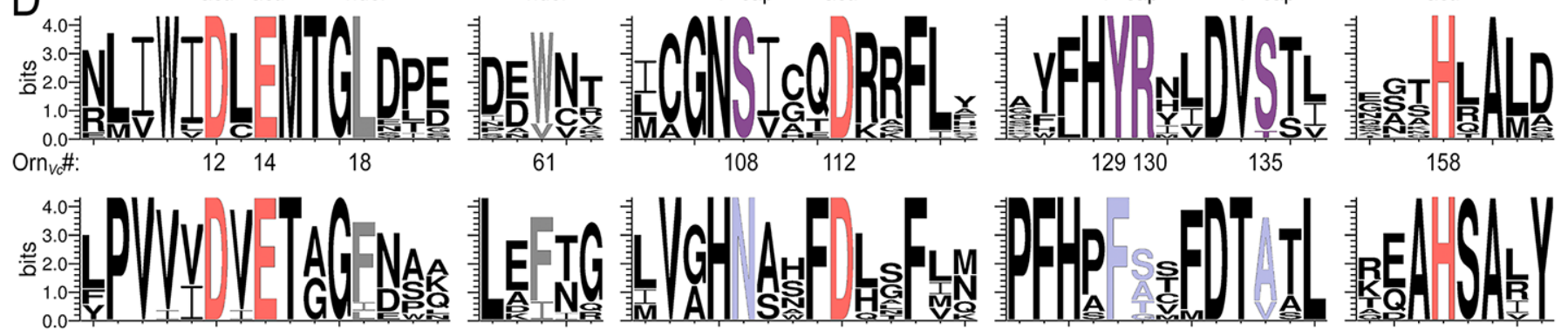

$\mathrm{RNaseT}_{E C} \#$ :

2325

29

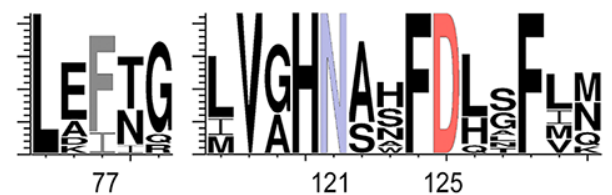

77

121

125

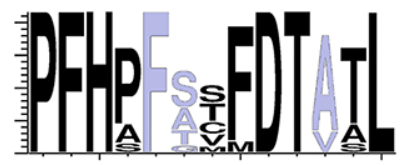

146147

152

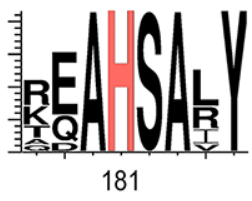

401

402

Figure 2. Structures reveal Orn's conserved substrate preference for diribonucleotides. Crystal structures of pGpG-bound V. cholerae Orn (A) and human

403 REXO2 (B) are shown in comparison to $E$. coli RNase T bound to substrate (PDB $3 n h 1 ;{ }^{33}$ )

404 (C), another DnaQ-fold 3'-5' exoribonuclease with a $\operatorname{DEDD}(\mathrm{h})$ active site motif. The top panels show ribbon representations of the dimeric enzymes. The insets are surface representations of the enzymes' active sites shown in similar orientations. The bottom panel describes the active site residues involved in RNA binding and catalysis. Residue numbering for REXO2 refers to its cytosolic isoform lacking the mitochondria-targeting pre-sequence. The sequence logos in (D) were constructed based on multi-sequence alignments of Orn and RNase T orthologs. Overall sequence identity ranges from $43-70 \%$ for Orn and $46-69 \%$ for RNase T. Sequence identifiers are provided in Table S2. Sequence logos were plotted using WebLogo 51. Conserved residues of the active site's DEDD motif ('cat.'; red), for ribonucleotide base binding ('nuc.'; grey), and of the phosphate cap ('P-cap'; purple) are highlighted. 
A

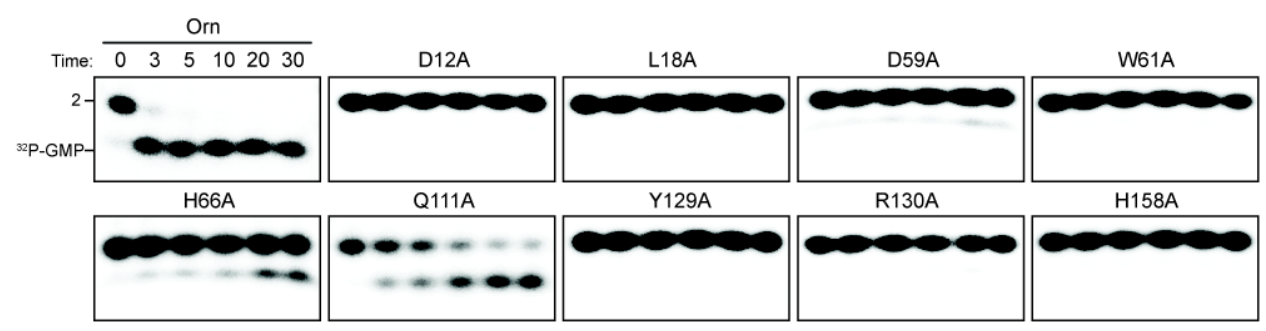

B

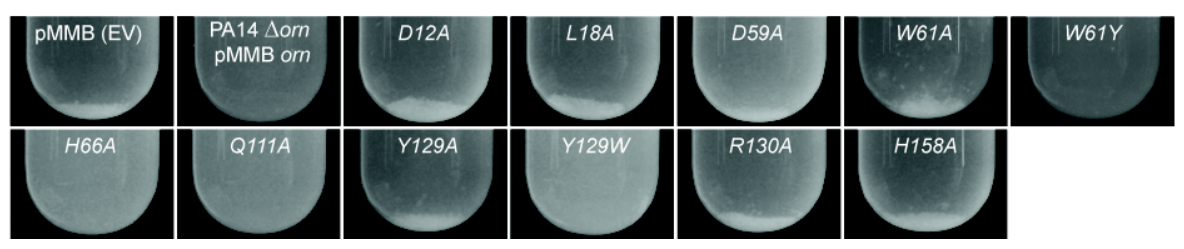

Figure 3 (Supplement to Figure 1A). Functional importance of active-site and phosphate-cap residues for Orn function. (A) In vitro enzyme activity. Degradation of ${ }^{32} \mathrm{P}$ pGpG $(1 \mu \mathrm{M})$ by purified wild-type Orn $v_{c}$ or variants with alanine substitutions $(5 \mathrm{nM})$ at the indicated sites was assessed. Samples were stopped the indicated times and analyzed by $20 \%$ Urea PAGE. Representative gel images are shown with the indicated RNA size. The graphs show means and SD of three independent experiments. (B) In vivo activity of alanine substituted orn $_{V c}$ alleles to complement $P$. aeruginosa $\Delta$ orn. Overnight cultures of the indicated strains were allowed to stand for 30 minutes without agitation to allow bacterial aggregates to sediment. Representative images of the cultures of triplicated assays are shown. 
430 431
A



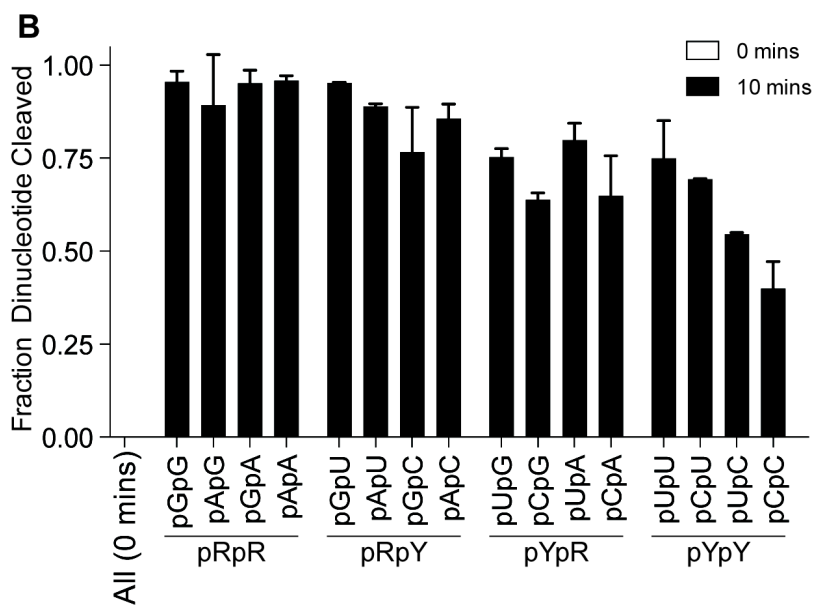

Figure 4 (Supplement to Figures 1 and S2B). Orn $v_{c}$ cleaves all diribonucleotides. (A) Orn $_{v_{c}}(5 n M)$ was incubated with di-purine $(p R p R)$, purine-pyrimidine (pRpY or pYpR), or dipyrimidine $(p Y p Y)$ diribonucleotides $(1 \mu \mathrm{M})$ containing the corresponding ${ }^{32} \mathrm{P}$-labeled RNA tracer. Aliquots of each reaction were stopped at the indicated times, and assessed by ureadenaturing 20\% polyacrylamide gel electrophoresis. (B) Quantification of the intensities of bands corresponding to the amount of diribonucleotide cleaved at the 10 min timepoint. Results are the average and SD of duplicate independent experiments. Orn cleaves all diribonucleotides to nucleoside monosphosphates, albeit to varying extents. Diribonucleotides consisting of two purines (pRpR) were hydrolyzed most efficiently, with over $90 \%$ of starting RNAs processed by 10 minutes. A majority $(>75 \%)$ of diribonucleotides with a $5^{\prime}$ purine (pRpY) were also processed by the 10-minute endpoint. However, diribonucleotides with a 5' pyrimidines exhibited moderately reduced levels of cleavage. Dipyrimidine ( $\mathrm{pYpY}$ ) substrates, and in particular $\mathrm{pUpC}$ and $\mathrm{pCpC}$, showed the slowest turnover from the substrates tested. These results demonstrate that while all diribonucleotides are acceptable substrates for Orn, the enzyme is likely to exhibit moderate preferences for diribonucleotides that contain a 5' purine. 


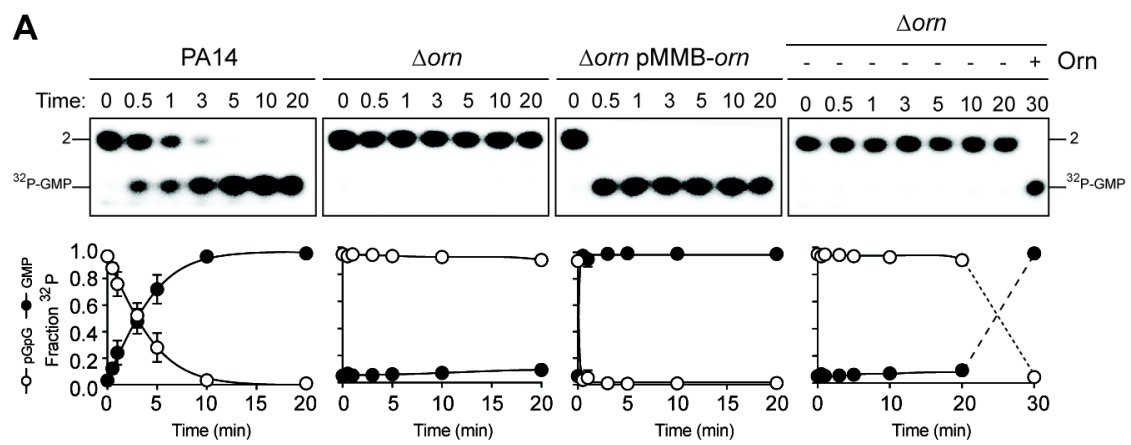

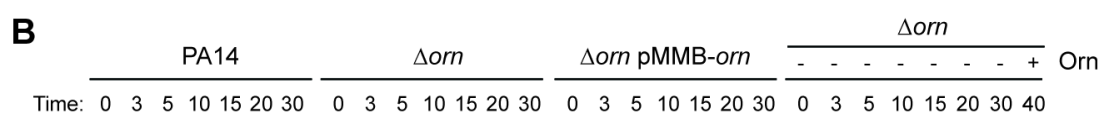
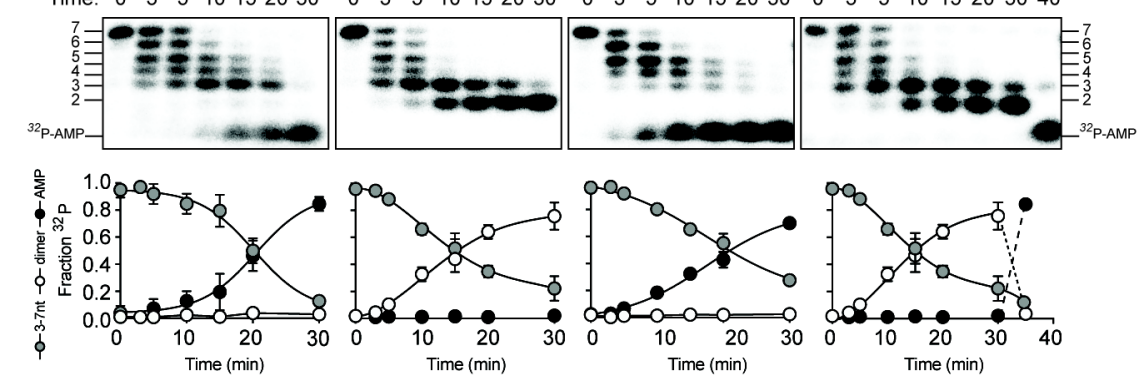

452 Figure 5. Orn acts as a diribonucleotidase in cell lysates. Degradation of ${ }^{32} \mathrm{P}-\mathrm{GG}(\mathrm{A})$ and with orn, or with $100 \mathrm{nM}$ of purified Orn. Samples were stopped at the indicated time and analyzed by $20 \%$ Urea PAGE. Representative gel images of triplicated assays are shown with the indicated RNA size. Graphs show quantitation of triplicate data for indicated RNA species over time. 
A
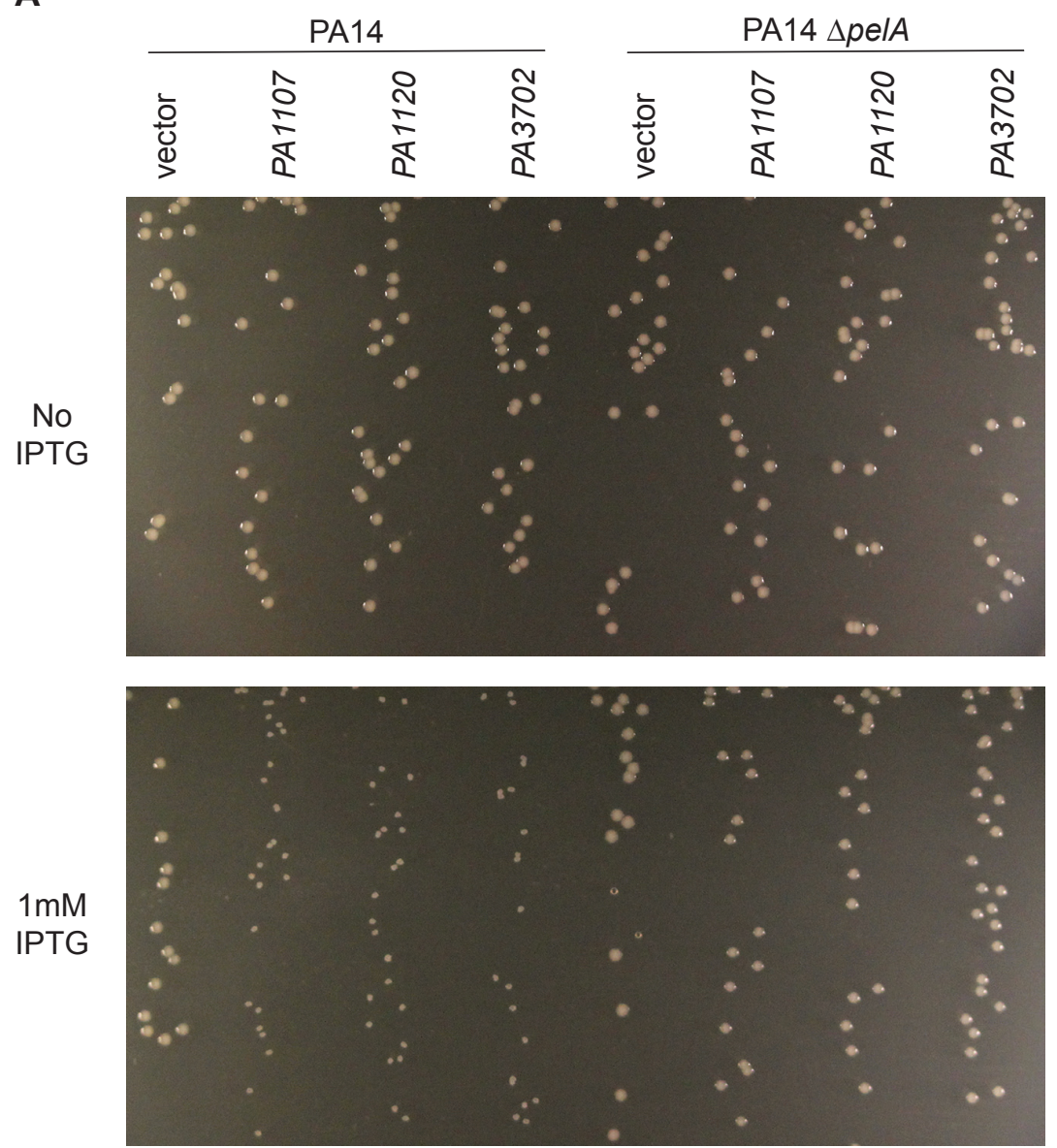

B


461

462

463

464

465

466

467

468
Figure 6. SCV phenotype of $\triangle$ orn is independent of c-di-GMP signaling. Bacterial cultures were diluted, $10 \mu \mathrm{L}$ was spotted on LB agar plates with the indicated concentration of IPTG and drip to form parallel lines of bacteria. After overnight incubation, representative images of the plates were taken of the plates are shown for (A) PA14 and PA14 $\Delta$ pelA harboring pMMB vector with the indicated diguanylate cyclase gene and (B) PA14 $\Delta$ orn and PA14 $\triangle$ orn $\triangle$ pelA-G with pMMB and pMMB-orn. Experiments were performed in triplicate. 


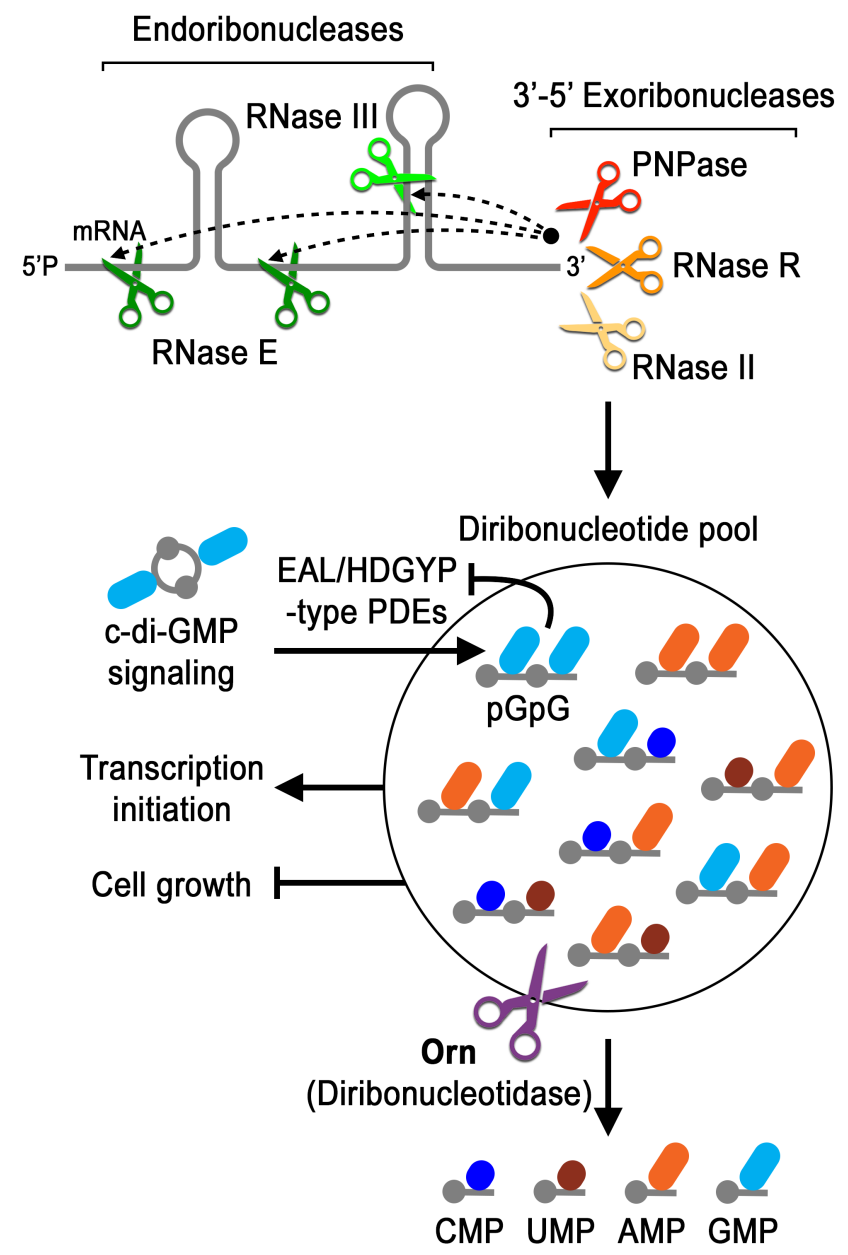

Figure 7. Model for Orn's cellular function as a diribonucleotidase. RNA degradation is initiated by fragmentation via endoribonucleases (e.g. RNase E and RNase III) that cleave unstructured or structured RNA sequences. RNA fragments are processed further at their 3' termini by 3'-5' exoribonucleases (e.g. PNPase, RNase R, and RNase II). Their combined activity produces the various diribonucleotides from the original RNA substrate. The pGpG (GG) linear diribonucleotide is also produced by linearization of c-di-GMP by specific phosphodiesterases, EAL- and HD-GYP-domain-containing enzymes, which terminate c-diGMP signaling. In Pseudomonas aeruginosa and likely other organism that rely on Orn for growth, Orn is the only diribonucleotidase that cleaves diribonucleotides to mononucleotides. In the absence of Orn, diribonucleotides accumulate with a drastic impact on cellular physiology, ranging from transcriptional changes, small-colony phenotypes and growth arrest, depending on the organism. Orn is also unique because it acts as the second phosphodiesterase in the degradation of c-di-GMP by clearing the pGpG intermediate. In an orn mutant, c-di-GMP levels are elevated through feedback inhibition of the c-di-GMPdegrading phosphodiesterases by $\mathrm{pGpG}$, leading to the associated biofilm phenotypes. 

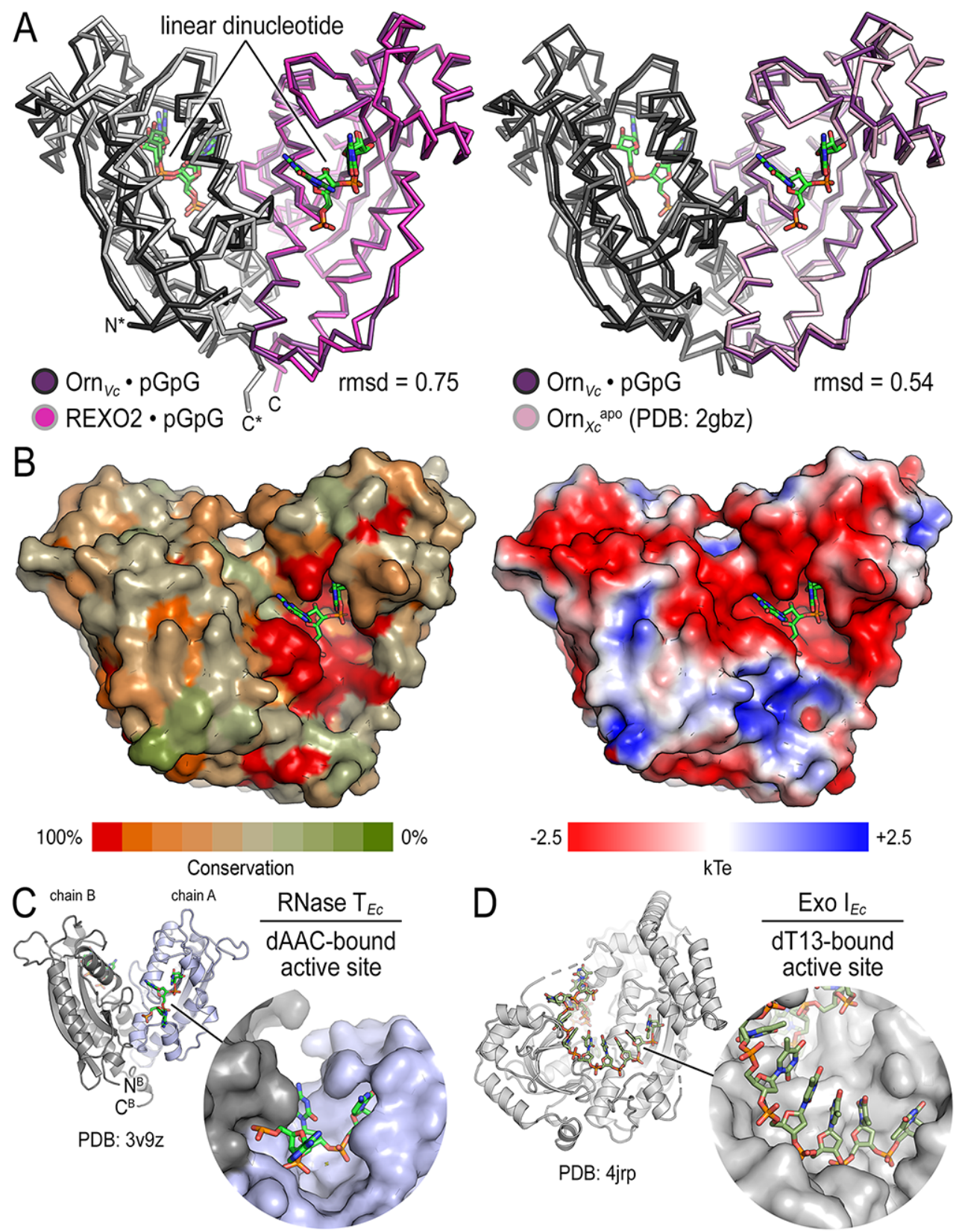

490

491

492

493

494

495

496

497

498

499

500

501
Figure S1 (Supplement to Figure 2). Structural comparison of Orn orthologs, RNase T and Exo I. (A) Superposition of Orn $v_{c}$ with REXO2 (left) and the substrate-free state of Orn from Xanthomonas campestris (PDB 2gbz; ${ }^{31}$ ) (right). Rmsd values are reported for the alignment of a monomer. (B) Surface properties of Orn enzymes. An alignment of Orn orthologs was used to map conservation scores onto the solvent accessible surface of Orn $V_{c}$ (left). High degree of conservation overlaps with the acidic active site observed in the electrostatic potential map calculated with the APBS software ${ }^{52}$ and based on Orn $V_{c}$ (right). (C) Active-site view of E. coli RNase T bound to dT13 (PDB 3v9z; ${ }^{32}$ ). (D) Active-site view of E. coli Exo I bound to dAAC (PDB 4jrp; ${ }^{34}$ ). 
bioRxiv preprint doi: https://doi.org/10.1101/546648; this version posted February 11,2019 . The copyright holder for this preprint (which was not certified by peer review) is the author/funder. All rights reserved. No reuse allowed without permission.

\begin{tabular}{|c|c|c|c|c|c|c|c|c|c|c|}
\hline Protein & OrnVc & OrnVc & OrnVc & OrnVc & OrnVc & OrnVc & OrnVc & REXO2 & REXO2 & REXO2 \\
\hline Substrate & pGpG & pApA & pApG & pGpA & pGpC & $\mathrm{pCpG}$ & $\mathrm{pCpU}$ & pGpG & pApA & pApG \\
\hline \multicolumn{11}{|l|}{ Data collection } \\
\hline Wavelength & 0.977 & 0.977 & 0.977 & 0.977 & 0.977 & 0.977 & 0.977 & 0.977 & 0.977 & 0.977 \\
\hline Resolution range & $\begin{array}{c}29.4-1.5 \\
(1.56-1.50)\end{array}$ & $\begin{array}{c}23.7-1.62 \\
(1.68-1.62) \\
\end{array}$ & $\begin{array}{c}38.9-1.53 \\
(1.59-1.53) \\
\end{array}$ & $\begin{array}{c}47.3-1.58 \\
(1.64-1.58) \\
\end{array}$ & $\begin{array}{cl}29.0 & -1.74 \\
(1.80 & -1.74) \\
\end{array}$ & $\begin{array}{cl}32.4 & -2.02 \\
(2.09 & -2.02) \\
\end{array}$ & $\begin{array}{cl}35.8 & -1.76 \\
(1.82 & -1.76) \\
\end{array}$ & $\begin{array}{c}41.2-1.43 \\
(1.48-1.43) \\
\end{array}$ & $\begin{array}{c}25.3-1.32 \\
(1.36-1.32) \\
\end{array}$ & $\begin{array}{cc}26.4 & -1.42 \\
(1.47 & -1.42) \\
\end{array}$ \\
\hline Space group & P 3221 & P 3221 & P 3221 & P 3221 & P 3221 & P 3221 & P 3221 & P 212121 & P 212121 & P 212121 \\
\hline Unit cell $a / b / c(\AA)$ & $\begin{array}{c}89.989 .9 \\
59.7 \\
\end{array}$ & $\begin{array}{c}89.489 .4 \\
59.5 \\
\end{array}$ & $\begin{array}{c}89.789 .7 \\
59.5 \\
\end{array}$ & $\begin{array}{c}89.689 .6 \\
59.7 \\
\end{array}$ & $\begin{array}{c}88.788 .7 \\
59.8 \\
\end{array}$ & $\begin{array}{c}89.089 .0 \\
59.9 \\
\end{array}$ & $\begin{array}{c}89.189 .1 \\
60.1 \\
\end{array}$ & $\begin{array}{c}45.887 .3 \\
123.8 \\
\end{array}$ & $\begin{array}{c}45.987 .3 \\
124.2 \\
\end{array}$ & $\begin{array}{c}45.887 .4 \\
124.3\end{array}$ \\
\hline Unit cell $\alpha / \beta / \gamma\left({ }^{\circ}\right)$ & 9090120 & 9090120 & 9090120 & 9090120 & 9090120 & 9090120 & 9090120 & 909090 & 909090 & 909090 \\
\hline Total reflections & $\begin{array}{l}533,852 \\
(43,456) \\
\end{array}$ & $\begin{array}{l}424,370 \\
(37,400) \\
\end{array}$ & $\begin{array}{l}499,153 \\
(43,484) \\
\end{array}$ & $\begin{array}{l}324,122 \\
(30,893)\end{array}$ & $\begin{array}{l}341,973 \\
(31,464) \\
\end{array}$ & $\begin{array}{l}220,944 \\
(20,997) \\
\end{array}$ & $\begin{array}{l}337,101 \\
(32,497) \\
\end{array}$ & $\begin{array}{l}697,560 \\
(42,227) \\
\end{array}$ & $\begin{array}{l}876,046 \\
(45,744) \\
\end{array}$ & $\begin{array}{l}754,926 \\
(64,979) \\
\end{array}$ \\
\hline Unique reflections & $\begin{array}{l}44,704 \\
(4,399) \\
\end{array}$ & $\begin{array}{l}35,164 \\
(3,462) \\
\end{array}$ & $\begin{array}{l}41,593 \\
(4,056) \\
\end{array}$ & $\begin{array}{l}38,242 \\
(3,736) \\
\end{array}$ & $\begin{array}{l}28,376 \\
(2,753) \\
\end{array}$ & $\begin{array}{l}18,318 \\
(1,797) \\
\end{array}$ & $\begin{array}{r}27,773 \\
(2,754) \\
\end{array}$ & $\begin{array}{l}91,370 \\
(8,272) \\
\end{array}$ & $\begin{array}{r}118,552 \\
(11,359) \\
\end{array}$ & $\begin{array}{l}95,178 \\
(9,301) \\
\end{array}$ \\
\hline Multiplicity & $11.9(9.9)$ & $12.1(10.8)$ & $12.0(10.7)$ & $8.5(8.3)$ & $12.1(11.4)$ & $12.1(11.7)$ & $12.1(11.8)$ & $7.6(5.1)$ & $7.4(4.0)$ & $7.9(7.0)$ \\
\hline Completeness (\%) & $99.9(99.6)$ & $99.9(99.3)$ & $99.8(98.2)$ & $99.7(98.0)$ & $99.8(98.3)$ & $99.9(99.7)$ & $100.0(99.9)$ & $99.0(91.0)$ & $99.6(96.8)$ & $99.8(98.6)$ \\
\hline I/sigma(I) & $18.2(1.7)$ & $29.4(1.9)$ & $21.4(2.0)$ & $18.7(1.9)$ & $25.9(1.9)$ & $22.2(2.1)$ & $22.6(2.2)$ & $13.6(1.5)$ & $17.8(1.7)$ & $16.5(2.2)$ \\
\hline Wilson B-factor & 15.98 & 26.32 & 18.37 & 22.94 & 30.18 & 37.21 & 30.56 & 12.17 & 12.62 & 14.23 \\
\hline CC1/2 & $1.00(0.66)$ & $1.00(0.71)$ & $1.00(0.75)$ & $1.00(0.70)$ & $1.00(0.71)$ & $1.00(0.77)$ & $1.00(0.76)$ & $1.00(0.56)$ & $1.00(0.75)$ & $1.00(0.80)$ \\
\hline \multicolumn{11}{|l|}{ Refinement } \\
\hline Reflections (R-free) & $2,236(220)$ & $1,762(177)$ & $2,081(201)$ & $1,916(188)$ & $1,421(137)$ & $916(81)$ & $1,390(137)$ & $2,275(206)$ & $2,958(284)$ & $2,371(230)$ \\
\hline R-work & 0.1467 & 0.1461 & 0.1452 & 0.1578 & 0.1545 & 0.1621 & 0.1605 & 0.1583 & 0.1528 & 0.1538 \\
\hline$R$-free & 0.1601 & 0.1546 & 0.1579 & 0.1646 & 0.1698 & 0.1875 & 0.1835 & 0.1782 & 0.1702 & 0.1725 \\
\hline Non-hydrogen atoms & 1,861 & 1,802 & 1,843 & 1,782 & 1,778 & 1,708 & 1,737 & 3,704 & 3,862 & 3,929 \\
\hline macromolecules & 1,553 & 1,533 & 1,534 & 1,515 & 1,551 & 1,549 & 1,526 & 3,214 & 3,338 & 3,364 \\
\hline ligands & 1 & 1 & 1 & 1 & 1 & 1 & 1 & 29 & 29 & 9 \\
\hline solvent & 307 & 268 & 308 & 266 & 226 & 158 & 210 & 461 & 495 & 556 \\
\hline Protein residues & 181 & 181 & 181 & 181 & 180 & 181 & 181 & 369 & 381 & 381 \\
\hline Rms (bonds) & 0.005 & 0.006 & 0.006 & 0.006 & 0.005 & 0.006 & 0.006 & 0.006 & 0.005 & 0.005 \\
\hline Rms (angles) & 0.84 & 0.82 & 0.84 & 1.15 & 0.84 & 0.84 & 0.85 & 0.81 & 0.83 & 0.83 \\
\hline \multicolumn{11}{|l|}{ Ramachandran } \\
\hline favored (\%) & 98.32 & 99.44 & 98.88 & 98.32 & 100.00 & 98.88 & 99.44 & 99.18 & 99.20 & 98.94 \\
\hline allowed (\%) & 1.68 & 0.56 & 1.12 & 1.68 & 0.00 & 1.12 & 0.56 & 0.82 & 0.80 & 1.06 \\
\hline outliers (\%) & 0.00 & 0.00 & 0.00 & 0.00 & 0.00 & 0.00 & 0.00 & 0.00 & 0.00 & 0.00 \\
\hline Rotamer outliers (\%) & 0.00 & 0.00 & 0.00 & 0.00 & 0.00 & 0.60 & 0.61 & 0.00 & 0.27 & 0.00 \\
\hline Clashscore & 1.94 & 1.32 & 0.99 & 1.00 & 1.31 & 2.60 & 2.32 & 1.08 & 1.04 & 1.78 \\
\hline Average B-factor & 21.83 & 32.63 & 24.51 & 29.40 & 36.37 & 43.95 & 36.31 & 18.45 & 19.57 & 22.59 \\
\hline macromolecules & 19.18 & 30.20 & 21.73 & 27.15 & 34.67 & 43.14 & 34.80 & 16.60 & 17.67 & 20.33 \\
\hline ligands & 14.60 & 24.65 & 16.01 & 20.33 & 31.09 & 48.74 & 48.05 & 33.16 & 31.27 & 55.54 \\
\hline solvent & 35.24 & 46.55 & 38.37 & 42.28 & 48.09 & 51.82 & 47.28 & 30.42 & 31.70 & 35.68 \\
\hline
\end{tabular}


bioRxiv preprint doi: https://doi.org/10.1101/546648; this version posted February 11,2019 . The copyright holder for this preprint (which was not certified by peer review) is the author/funder. All rights reserved. No reuse allowed without permission.

504

Table S2. Sequences used to calculate surface conservation and generate Weblogos.

\begin{tabular}{|c|c|c|c|c|c|}
\hline \multicolumn{3}{|c|}{ Oligoribonuclease } & \multicolumn{3}{|c|}{ RNase T } \\
\hline Strain & accession\# & $\% I D$ to Orn $_{v c}{ }^{b}$ & Strain & accession\# $\#^{a}$ & \%ID to RNaseT ${ }_{V c}^{b}$ \\
\hline Vibrio cholerae & Q9KV17 & 100 & Vibrio cholerae & Q9KT97 & 100 \\
\hline Escherichia coli & P0A784 & 68 & Escherichia coli & P30014 & 69 \\
\hline Coxiella burnetii & Q83C93 & 59 & Coxiella burnetii & Q83B16 & 46 \\
\hline Haemophilus influenzae & P45340 & 64 & Haemophilus influenzae & P44639 & 59 \\
\hline Acinetobacter baumannii & V5VGJ9 & 59 & Acinetobacter baumannii & DOCDY9 & 53 \\
\hline Xanthomonas campestris & Q8P8S1 & 55 & Xanthomonas campestris & Q8PAG3 & 47 \\
\hline Pseudomonas aeruginosa & P57665 & 70 & Pseudomonas aeruginosa & Q9HY82 & 63 \\
\hline Pseudomonas fluorescens & Q3KIZ7 & 69 & Pseudomonas fluorescens & Q3K7J7 & 61 \\
\hline Azotobacter vinelandii & C1DLP5 & 66 & Azotobacter vinelandii & C1DQV0 & 65 \\
\hline Shigella flexneri & Q83IJ9 & 67 & Shigella flexneri & Q0T4C2 & 69 \\
\hline Legionella pneumophila & Q5ZRY0 & 62 & Legionella pneumophila & Q5ZRB8 & 52 \\
\hline Mycobacterium tuberculosis & P9WIU1 & 43 & & & \\
\hline Streptomyces coelicolor & P57666 & 51 & & & \\
\hline Homo sapiens & Q9Y3B8 & 50 & & & \\
\hline
\end{tabular}

${ }^{\mathrm{a}}$ : Based on Uniprot database (1); : Based on ClustalOmega multi-sequence alignment scores (2).

505

506

507

508

509

\section{References}

510

511

1: The UniProt Consortium. (2017). UniProt: the universal protein knowledgebase. Nucleic Acids Res.

512 45(D1): D158-D169.

513

514 2: Sievers, F., Wilm, A., Dineen, D., Gibson, T.J., Karplus, K., Li, W., Lopez, R., McWilliam, H., Remmert, 515 M., Söding, J., Thompson, J.D., Higgins, D.G. (2011). Fast, scalable generation of high-quality protein 516 multiple sequence alignments using Clustal Omega. Mol Syst Biol. 7:539. 



Figure S2 (Supplement to Figures $2 A$ and B). Structural comparison of Orn $v_{v c}$ and REXO2 bound to diverse diribonucleotides. (A) Crystal structures of REXO2 bound to pApA and pApG. The structural overview (left) shows a C-terminal helix that is unique to Orn orthologs in higher eukaryotes. (B) Crystal structures of Orn $_{v c}$ bound to di-purine (pApA, pApG, pGpA), purine-pyrimidine ( $p G p C, p C p G)$, and di-pyrimidine ( $p C p U)$ substrates. Views are identical to those shown in Figure 2 for the REXO2:pGpG and Orn:pGpG complexes. 
A

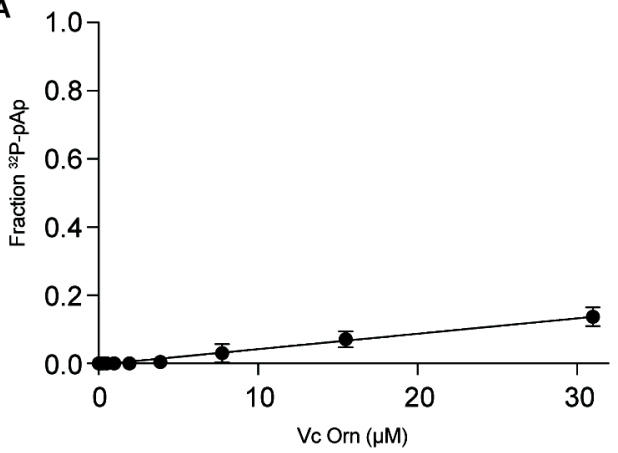

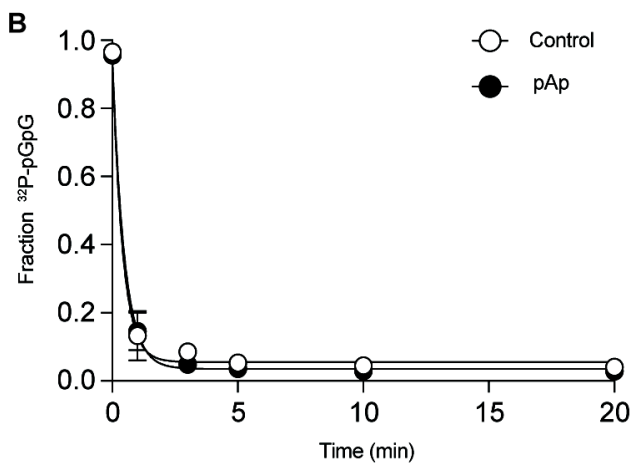

Figure S3. pAp has no effect on Orn activity. $(A)$ The dissociation constant $\left(K_{d}\right)$ of ${ }^{32} \mathrm{P}$ pAp binding to Orn. $K_{d}$ could not be measured as ${ }^{32} \mathrm{P}-\mathrm{pAp}$ binding to Orn was not saturatable. (B) The rate of ${ }^{32} \mathrm{P}-\mathrm{pG}$ gG degradation by Orn in the absence or presence of unlabeled $\mathrm{pAp}$ $(100 \mathrm{mM})$. All data shown represent duplicate independent experiments. 
bioRxiv preprint doi: https://doi.org/10.1101/546648; this version posted February 11,2019 . The copyright holder for this preprint (which was not certified by peer review) is the author/funder. All rights reserved. No reuse allowed without permission.

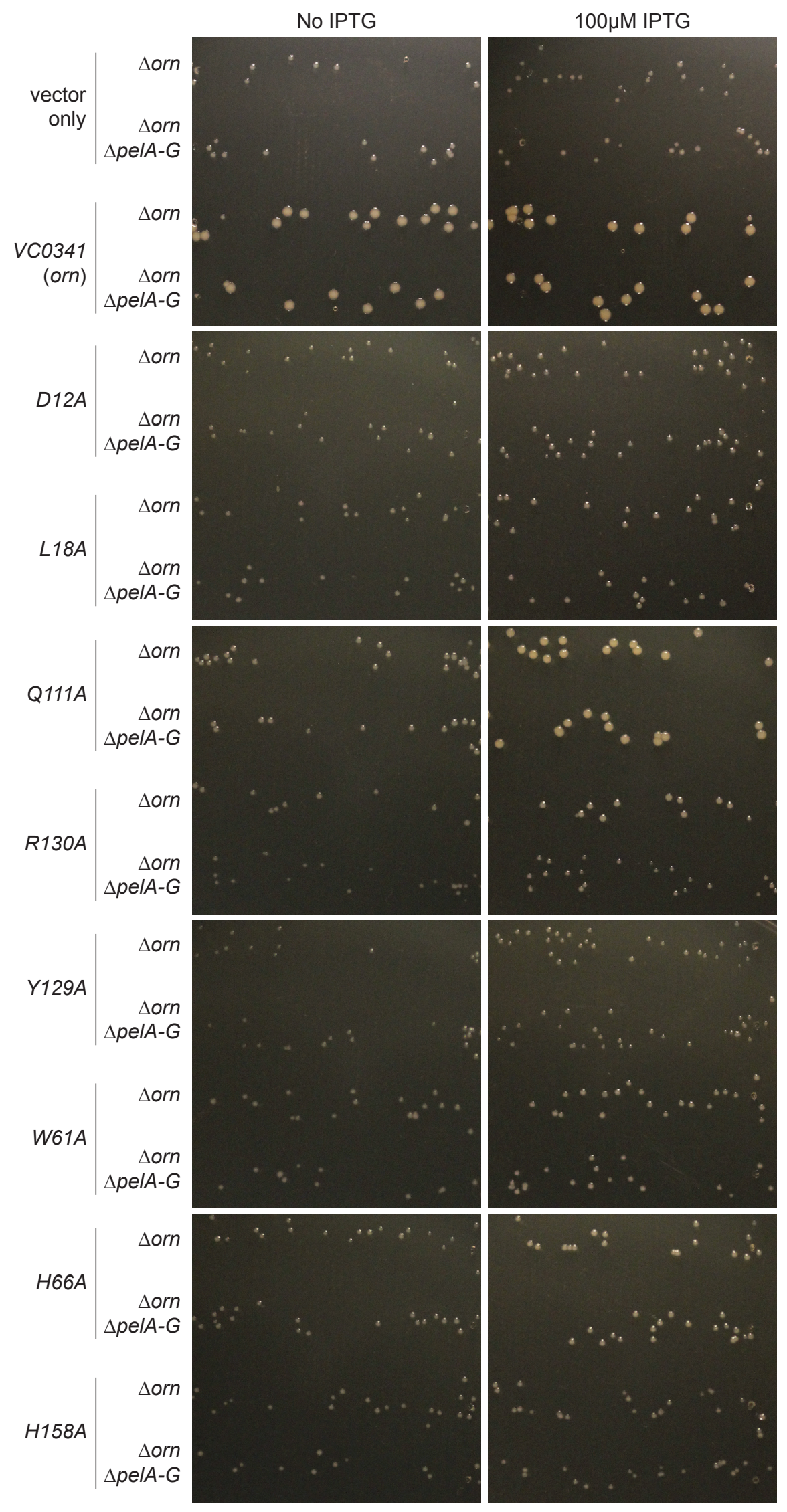


538 Figure S4. SCV phenotype of $\Delta$ orn is complemented by functional orn alleles. Bacterial 539 cultures were diluted, $10 \mu \mathrm{L}$ was spotted on LB agar plates with the indicated concentration of 540 IPTG and drip to form parallel lines of bacteria. After overnight incubation, representative 541 images of the plates were taken of PA14 $\Delta$ orn and PA14 $\Delta$ orn $\Delta$ pelA-G with pMMB alone or 542 containing the indicated allele of orn. Experiments were performed in triplicate. 
1 Hui, M. P., Foley, P. L. \& Belasco, J. G. Messenger RNA degradation in bacterial cells. Annu Rev Genet 48, 537-559, doi:10.1146/annurev-genet-120213-092340 (2014). Biol 10, 627-635, doi:10.4161/rna.24393 (2013). multienzyme process in vivo. Cell 86, 503-512 (1996). pathway. Proc Natl Acad Sci U S A 96, 4372-4377 (1999). Palace, S. G., Proulx, M. K., Lu, S., Baker, R. E. \& Goguen, J. D. Genome-wide mutant fitness profiling identifies nutritional requirements for optimal growth of Yersinia pestis in deep tissue. MBio 5, doi:10.1128/mBio.01385-14 (2014). Kamp, H. D., Patimalla-Dipali, B., Lazinski, D. W., Wallace-Gadsden, F. \& Camilli, A. Gene fitness landscapes of Vibrio cholerae at important stages of its life cycle. PLoS pathogens $\mathbf{9}$, e1003800, doi:10.1371/journal.ppat.1003800 (2013). substrate specificity. J Biol Chem 277, 29654-29661, doi:10.1074/jbc.M204252200 (2002). exoribonuclease RNase R. Comparison with RNase II. J Biol Chem 277, 21624-21629, doi:10.1074/jbc.M202942200 (2002). Cheng, Z. F. \& Deutscher, M. P. An important role for RNase R in mRNA decay. Mol Cell 17, 313-318, doi:10.1016/j.molcel.2004.11.048 (2005).

10 Frazao, C. et al. Unravelling the dynamics of RNA degradation by ribonuclease II and its RNAbound complex. Nature 443, 110-114, doi:10.1038/nature05080 (2006).

11 Krasteva, P. V. \& Sondermann, H. Versatile modes of cellular regulation via cyclic dinucleotides. Nature chemical biology 13, 350-359, doi:10.1038/nchembio.2337 (2017). bacterial second messenger. Microbiol. Mol. Biol. Rev. 77, 1-52, doi:10.1128/MMBR.00043-12 (2013). Hengge, R. Principles of c-di-GMP signalling in bacteria. Nat Rev Microbiol 7, 263-273 (2009). Jenal, U., Reinders, A. \& Lori, C. Cyclic di-GMP: second messenger extraordinaire. Nat Rev Microbiol 15, 271-284, doi:10.1038/nrmicro.2016.190 (2017).

Ross, P. et al. Regulation of cellulose synthesis in Acetobacter xylinum by cyclic diguanylic acid. Nature 325, 279-281 (1987).

Tal, R. et al. Three cdg operons control cellular turnover of cyclic di-GMP in Acetobacter xylinum: genetic organization and occurrence of conserved domains in isoenzymes. $J$ Bacteriol 180, 4416-4425 (1998). hydrolase domain implicated in signal transduction. Journal of molecular microbiology and biotechnology 1, 303-305 (1999).

8 Cohen, D. et al. Oligoribonuclease is a central feature of cyclic diguanylate signaling in Pseudomonas aeruginosa. Proc Natl Acad Sci U S A 112, 11359-11364, doi:10.1073/pnas.1421450112 (2015). 
19 Orr, M. W. et al. Oligoribonuclease is the primary degradative enzyme for $\mathrm{pGpG}$ in Pseudomonas aeruginosa that is required for cyclic-di-GMP turnover. Proc Natl Acad Sci U S A 112, E5048-5057, doi:10.1073/pnas.1507245112 (2015).

20 Stevens, A. \& Niyogi, S. K. Hydrolysis of oligoribonucleotides by an enzyme fraction from Escherichia coli. Biochem Biophys Res Commun 29, 550-555 (1967).

21 Niyogi, S. K. \& Datta, A. K. A novel oligoribonuclease of Escherichia coli. I. Isolation and properties. J Biol Chem 250, 7307-7312 (1975).

22 Datta, A. K. \& Niyogi, K. A novel oligoribonuclease of Escherichia coli. II. Mechanism of action. J Biol Chem 250, 7313-7319 (1975).

23 Yu, D. \& Deutscher, M. P. Oligoribonuclease is distinct from the other known exoribonucleases of Escherichia coli. J Bacteriol 177, 4137-4139 (1995).

24 Mechold, U., Ogryzko, V., Ngo, S. \& Danchin, A. Oligoribonuclease is a common downstream target of lithium-induced pAp accumulation in Escherichia coli and human cells. Nucleic Acids Res 34, 2364-2373, doi:10.1093/nar/gkl247 (2006).

25 Mechold, U., Fang, G., Ngo, S., Ogryzko, V. \& Danchin, A. Ytql from Bacillus subtilis has both oligoribonuclease and pAp-phosphatase activity. Nucleic Acids Res 35, 4552-4561, doi:10.1093/nar/gkm462 (2007).

26 Fang, M. et al. Degradation of nanoRNA is performed by multiple redundant RNases in Bacillus subtilis. Nucleic Acids Res 37, 5114-5125, doi:10.1093/nar/gkp527 (2009).

27 Liu, M. F. et al. Identification of a novel nanoRNase in Bartonella. Microbiology 158, 886-895, doi:10.1099/mic.0.054619-0 (2012).

28 Roelofs, K. G., Wang, J., Sintim, H. O. \& Lee, V. T. Differential radial capillary action of ligand assay for high-throughput detection of protein-metabolite interactions. Proc. Natl. Acad. Sci. U. S. A. 108, 15528-15533, doi:1018949108 [pii]

10.1073/pnas.1018949108 (2011).

29 Kwok, C. K. \& Merrick, C. J. G-Quadruplexes: Prediction, Characterization, and Biological Application. Trends Biotechnol 35, 997-1013, doi:10.1016/j.tibtech.2017.06.012 (2017).

30 Bruni, F., Gramegna, P., Oliveira, J. M., Lightowlers, R. N. \& Chrzanowska-Lightowlers, Z. M. REXO2 is an oligoribonuclease active in human mitochondria. PloS one 8, e64670, doi:10.1371/journal.pone.0064670 (2013).

31 Chin, K. H., Yang, C. Y., Chou, C. C., Wang, A. H. \& Chou, S. H. The crystal structure of XC847 from Xanthomonas campestris: a 3'-5' oligoribonuclease of DnaQ fold family with a novel opposingly shifted helix. Proteins 65, 1036-1040, doi:10.1002/prot.21148 (2006).

32 Hsiao, Y. Y., Duh, Y., Chen, Y. P., Wang, Y. T. \& Yuan, H. S. How an exonuclease decides where to stop in trimming of nucleic acids: crystal structures of RNase T-product complexes. Nucleic Acids Res 40, 8144-8154, doi:10.1093/nar/gks548 (2012).

33 Hsiao, Y. Y. et al. Structural basis for RNA trimming by RNase T in stable RNA 3'-end maturation. Nature chemical biology 7, 236-243, doi:10.1038/nchembio.524 (2011).

34 Korada, S. K. et al. Crystal structures of Escherichia coli exonuclease I in complex with singlestranded DNA provide insights into the mechanism of processive digestion. Nucleic Acids Res 41, 5887-5897, doi:10.1093/nar/gkt278 (2013).

35 Postic, G., Danchin, A. \& Mechold, U. Characterization of NrnA homologs from Mycobacterium tuberculosis and Mycoplasma pneumoniae. RNA (New York, N.Y 18, 155-165, doi:10.1261/rna.029132.111 (2012). 
63236 D'Argenio, D. A., Calfee, M. W., Rainey, P. B. \& Pesci, E. C. Autolysis and autoaggregation in

633

634 Pseudomonas aeruginosa colony morphology mutants. J Bacteriol 184, 6481-6489 (2002).

635

636

637

638

639

640

641

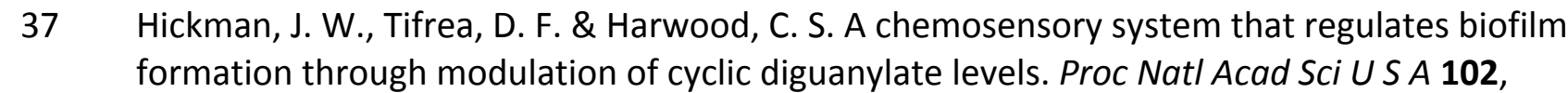
14422-14427 (2005).

38 Baraquet, C., Murakami, K., Parsek, M. R. \& Harwood, C. S. The FleQ protein from Pseudomonas aeruginosa functions as both a repressor and an activator to control gene expression from the pel operon promoter in response to c-di-GMP. Nucleic Acids Res 40, 7207-7218, doi:gks384 [pii]

10.1093/nar/gks384 (2012).

642

39 Matsuyama, B. Y. et al. Mechanistic insights into c-di-GMP-dependent control of the biofilm

643

644

645

646

647

648

649

650 regulator FleQ from Pseudomonas aeruginosa. Proc Natl Acad Sci U S A 113, E209-218, doi:10.1073/pnas.1523148113 (2016).

40 Lee, V. T. et al. A cyclic-di-GMP receptor required for bacterial exopolysaccharide production. Mol Microbiol 65, 1474-1484 (2007).

41 Kulasakara, H. et al. Analysis of Pseudomonas aeruginosa diguanylate cyclases and phosphodiesterases reveals a role for bis-(3'-5')-cyclic-GMP in virulence. Proc. Natl. Acad. Sci. U. S. A. 103, 2839-2844, doi:0511090103 [pii]

651

652

653

654

655

656

657

658

659

660 10.1073/pnas.0511090103 (2006).

42 Ueda, A. \& Wood, T. K. Connecting quorum sensing, c-di-GMP, pel polysaccharide, and biofilm formation in Pseudomonas aeruginosa through tyrosine phosphatase TpbA (PA3885). PLoS pathogens 5, e1000483, doi:10.1371/journal.ppat.1000483 (2009).

43 Malone, J. G. et al. YfiBNR mediates cyclic di-GMP dependent small colony variant formation and persistence in Pseudomonas aeruginosa. PLoS pathogens 6, e1000804, doi:10.1371/journal.ppat.1000804 (2010).

44 Goldman, S. R. et al. NanoRNAs prime transcription initiation in vivo. Mol Cell 42, 817-825, doi:S1097-2765(11)00419-9 [pii]

10.1016/j.molcel.2011.06.005 (2011).

45 Evans, P. Scaling and assessment of data quality. Acta Crystallogr D Biol Crystallogr 62, 72-82, doi:10.1107/S0907444905036693 (2006).

661

662

Kabsch, W. Integration, scaling, space-group assignment and post-refinement. Acta Crystallogr

663 D Biol Crystallogr 66, 133-144, doi:10.1107/S0907444909047374 (2010).

664

Adams, P. D. et al. PHENIX: a comprehensive Python-based system for macromolecular

665

666 structure solution. Acta Crystallogr D Biol Crystallogr 66, 213-221, doi:10.1107/S0907444909052925 (2010).

668

48 Emsley, P., Lohkamp, B., Scott, W. G. \& Cowtan, K. Features and development of Coot. Acta Crystallogr D Biol Crystallogr 66, 486-501, doi:10.1107/S0907444910007493 (2010). Morin, A. et al. Collaboration gets the most out of software. eLife 2, e01456, doi:10.7554/eLife.01456 (2013). Methods Enzymol 549, 489-512, doi:10.1016/B978-0-12-801122-5.00021-0 (2014). 
67552 Baker, N. A., Sept, D., Joseph, S., Holst, M. J. \& McCammon, J. A. Electrostatics of

676 nanosystems: application to microtubules and the ribosome. Proc Natl Acad Sci U S A 98,

677

678 10037-10041, doi:10.1073/pnas.181342398 (2001). 